\title{
Whole-body vibration training - better care for COPD patients: a systematic review and meta-analysis
}

This article was published in the following Dove Press journal: International Journal of COPD

\author{
Jian Zhou ${ }^{1,2, *}$ \\ Long Pang ${ }^{2, *}$ \\ Nan Chen ${ }^{1,2}$ \\ Zihuai Wang ${ }^{1,2}$ \\ Chengdi Wang ${ }^{3}$ \\ Yang $\mathrm{Hai}^{1,2}$ \\ Mengyuan Lyu ${ }^{2,4}$ \\ Hongjin Lai ${ }^{2}$ \\ Feng Lin ${ }^{1,5}$
}

'Department of Thoracic Surgery, West China Hospital, Sichuan University, Chengdu, People's Republic of China; ${ }^{2}$ West China School of Medicine, Sichuan University, Chengdu, People's Republic of China; ${ }^{3}$ Department of Respiratory Medicine, Sichuan University, Chengdu, People's Republic of China; ${ }^{4}$ Department of Laboratory Medicine, West China Hospital, Sichuan University, Chengdu, People's Republic of China; ${ }^{5}$ Western China Collaborative Innovation Center for Early Diagnosis and Multidisciplinary Therapy of Lung Cancer, Sichuan University, Chengdu, People's Republic of China

*These authors contributed equally to this work
Correspondence: Feng Lin Department of Thoracic Surgery, West China Hospital, Sichuan University, No 37, Guoxue Alley, Chengdu, Sichuan 61004I, People's Republic of China Tel +8628 85422494

Fax +86 2885422494

Email linfeng0220@aliyun.com
Purpose: Whole-body vibrating training (WBVT) is a modality aiming to improve neuromuscular performance of patients with COPD. However, a consensus on the effects of WBVT has not been reached. We aimed to clarify the effects of WBVT on functional exercise capacity, pulmonary function, and quality of life in COPD patients.

Patients and methods: PubMed, Web of Science, and EMBASE were searched through April 5, 2018. We calculated the pooled weight mean difference (WMD) using a random-effects model. Quality assessment and publication bias analyses were also performed.

Results: We included eight randomized control trials involving 365 patients. Compared with control group, WBVT increased 6-minute walking distance (6-MWD) (WMD: $62.14 \mathrm{~m}$; 95\% CI: 48.12-76.16; $P<0.001$ ), the change of 6-MWD ( $\Delta 6-\mathrm{MWD}$ ) (WMD: $42.33 \mathrm{~m}$; 95\% CI: $15.21-69.45 ; P=0.002)$, the change of the time to finish five repeated sit-to-stand tests (WMD: -2.07 seconds; $95 \%$ CI: -4.00 to $-0.05 ; P=0.04$ ), and decreased the change of St George's Respiratory Questionnaire score (WMD: -6.65 points; $95 \%$ CI: -10.52 to -2.78 ; $P<0.001)$. However, no significant difference was found between the two groups regarding forced expired volume in 1 second $\left(\mathrm{FEV}_{1}\right)$ (\% predicated), change of $\mathrm{FEV}_{1}$ ( $\%$ predicated), sit-to-stand test, 6-MWD (\% predicated), change of 6-MWD (\% predicated), St George's Respiratory Questionnaire score, COPD Assessment Test score, and change of COPD Assessment Test score.

Conclusion: WBVT has beneficial effects on functional exercise capacity for COPD patients. Keywords: COPD, whole-body vibration training, functional capacity, pulmonary rehabilitation

\section{Introduction}

Vibration is a mechanical stimulus, and the biomechanical variables that determine its intensity are the frequency and amplitude. Vibration training is the deliberate exposure to the body of varying frequencies using certain joint angles for any limited time. Vibration training is quite a new technology in sports science. Athletes and fitness and rehabilitation centers are widely using vibration training in their programs. Whole-body vibration training (WBVT) is one of the therapeutic pulmonary rehabilitation modalities aiming to improve neuromuscular performance of patients with neuromuscular dysfunction, which is applied through a vibration surface that generates sinusoidal vibrations. ${ }^{1}$ During WBVT, muscle contraction is elicited, and selected muscles can thereby be stimulated and strengthened. ${ }^{2}$

WBVT devices deliver vibrations across a range of frequencies $(15-60 \mathrm{~Hz})$ and displacements from $<1$ to $10 \mathrm{~mm}$. Numerous combinations of amplitudes and 
frequencies with current technology make it possible for a wide variety of WBVT protocols to be used on humans. Vibrating platforms fall into different, distinct categories. The type of platform used is a moderator of the effect and result of the training or therapy performed. It can be pivotal (vibrating from side to side) or lineal (vibrating up and down). When applied in patients with COPD, it usually employs low-amplitude, low-frequency mechanical stimulation.

Roelants et $\mathrm{al}^{3}$ reported that after a 24-week WBVT, the muscle strength and performance of elderly women increased to a similar extent as regular resistance training. Evidence suggested that WBVT is a promising training modality for patients with COPD. ${ }^{4}$ However, a more convincing conclusion is urgently needed.

Our study aimed to identify the effects of WBVT on improving functional exercise capacity, pulmonary function, and quality of life in COPD patients.

\section{Methods}

\section{Eligibility criteria}

For inclusion, the studies needed to 1 ) focus on patients with COPD; 2) compare the effects difference between WBVT and conventional training; 3) be randomized controlled trials (RCTs); and 4) be written in English. The studies were excluded if they met one of the following criteria: 1) were reviews, letters, conference abstracts, book chapters, animal experiments, and case reports; 2) essential information was incomplete; and 3) WBVT was not conducted for the purpose of improving patient's condition. Two independent reviewers determined study eligibility.

\section{Search strategy}

Two authors (JZ and LP) independently searched literatures in MEDLINE, EMBASE, and Web of Science through April 5, 2018 with the following search items: "COPD" chronic obstructive pulmonary disease "emphysema" or "chronic bronchitis" and "whole body vibration training" "vibration training" or "vibration." Then, manual searching of the reference lists of eligible studies was done to find potential relevant publications.

\section{Study selection}

Two independent reviewers (JZ and LP) screened the titles and abstracts of identified articles. The obtained full-text of potential articles were screened for further check.

\section{Data collection}

Data extraction was performed by two independent researchers ( $\mathrm{JZ}$ and LP), including publication information (the first author, country, publication year), patient characteristics (sample size, age, gender, disease, the grade of COPD), WBVT training regime (the intensity, the frequency, the duration), and compared outcomes (6-minute walking distance [6-MWD], forced expiratory volume in 1 second [FEV ${ }_{1}$, sit-to-stand test [SST], St George's Respiratory Questionnaire [SGRQ], COPD Assessment Test [CAT], Chronic Respiratory Questionnaire, Berg scale, and adverse events).

\section{Risk of bias in individual studies}

We assessed the risk of bias of included studies according the Risk of Bias Tool found in the Cochrane Handbook for Systemic Reviews of Interventions, ${ }^{5}$ with the following aspects: sequence generation and concealment of allocation (selection bias), blinding of participants and personnel (performance bias), blinding of outcome assessors (detection bias), incomplete outcome data addressed (attrition bias), free of selective reporting (reporting bias), and other bias. If any disagreements appeared, a third reviewer (FL) made a final decision.

\section{Sensitivity analyses and subgroup analyses}

We conducted sensitivity analyses to explore the potential reasons for the heterogeneity, by removing one included study each time and identifying the robustness of the results. Subgroups analyses were performed to find potential heterogeneity, including age ( $\geq 70$ or $<70$ years), ethnicity (European or American), the status of COPD (stable or severe), vibration type (side-alternating vibration or vertical vibration), and exercise duration $(\geq 1$ or $<1$ month).

\section{Synthesis of results}

The pooled outcomes were expressed as weighted mean difference (WMD) with $P$-value and 95\% CIs. The results were regarded statistically significant when $P<0.05$. Heterogeneity across studies was tested by using the $I^{2}$ statistic, which is a quantitative measure of inconsistency across studies. Studies were considered to have low heterogeneity when $I^{2}$ statistic was $25 \%-50 \%$, and those with an $I^{2}$ statistic $>75 \%$ were considered to have high heterogeneity. A random-effects model was used if the $I^{2}$ statistic $>50 \%$, otherwise the fixed-effect model was used. All statistical analyses were performed using Review Manager V.5.3 (The Cochrane Collaboration, Software Update, Oxford, UK). 


\section{Results \\ Study characteristics}

A total of 1,012 studies were identified by the initial database search. Only eight RCTs ${ }^{6-13}$ with 365 patients were included in our study after full evaluation (Figure 1). The included studies were published between 2012 and 2017. The analyzed patients were mainly the elderly with an average age varying from 58 to 75 years, community dwelling, and functionally independent. People with exacerbated COPD were studied in one trial. ${ }^{9}$ Other trials studied individuals with stable COPD. Generally, no special intervention was performed in a control group, but three trials performed physiotherapy, ${ }^{9}$ calisthenics training, ${ }^{12}$ and conventional resistance training, ${ }^{11}$ respectively. The primary outcomes included 6-MWD, $\mathrm{FEV}_{1}$, SST, SGRQ, CAT, CRQ, Berg scale, and the changes in these scores. Table 1 shows the study characteristics.

\section{Methodological quality of included studies}

The risk of the included studies was found to be acceptable. Three studies ${ }^{7,10,12}$ did not report the concealment of allocation. Due to the intervention (WBVT), it is not possible to blind patients or study personnel to the group allocation. All included studies did not report on any specific group being blinded in the articles, typically participants, personnel, and outcome assessors (Table 2 and Figure 2).

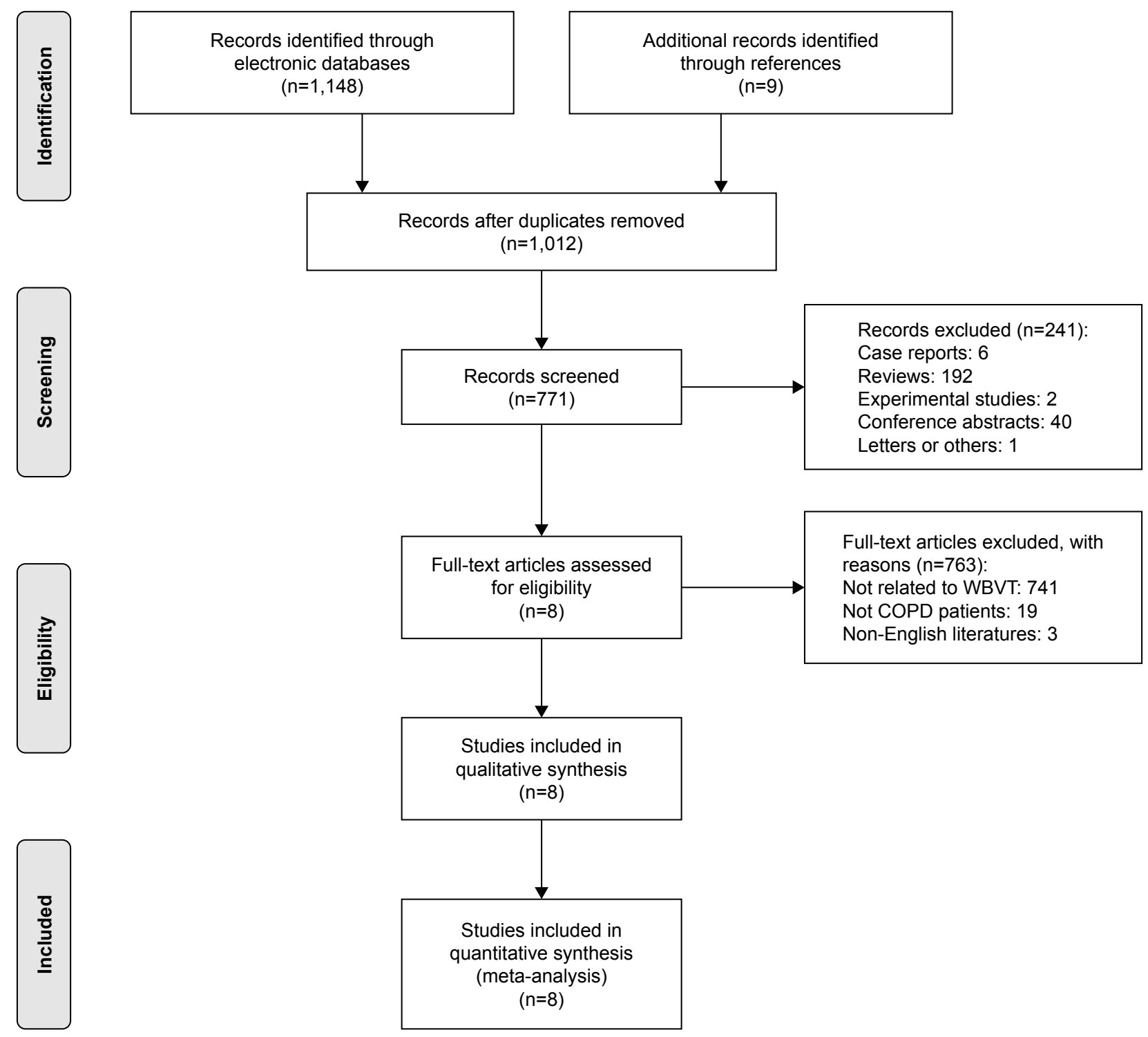

Figure I The PRISMA flow diagram of literature retrieval.

Note: Reproduced from Moher D, Liberati A, Tetzlaff J, Altman DG; The PRISMA Group (2009). Preferred Reporting Items for Systematic Reviews and Meta-Analyses: The PRISMA Statement. PLoS Med 6(7):el000097. doi:10.1371/journal.pmedI000097. ${ }^{36}$

Abbreviation: WBVT, whole-body vibration training. 


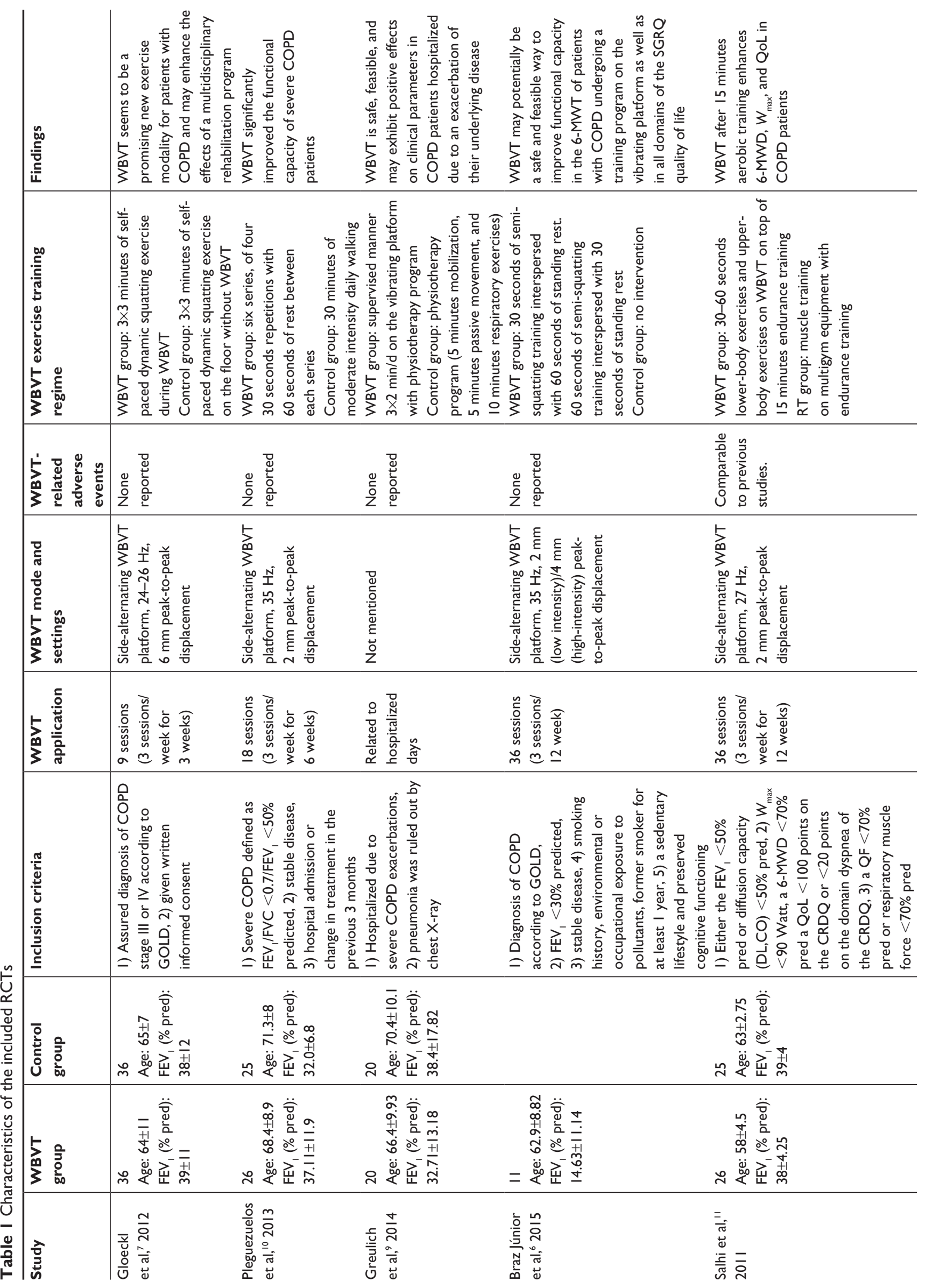




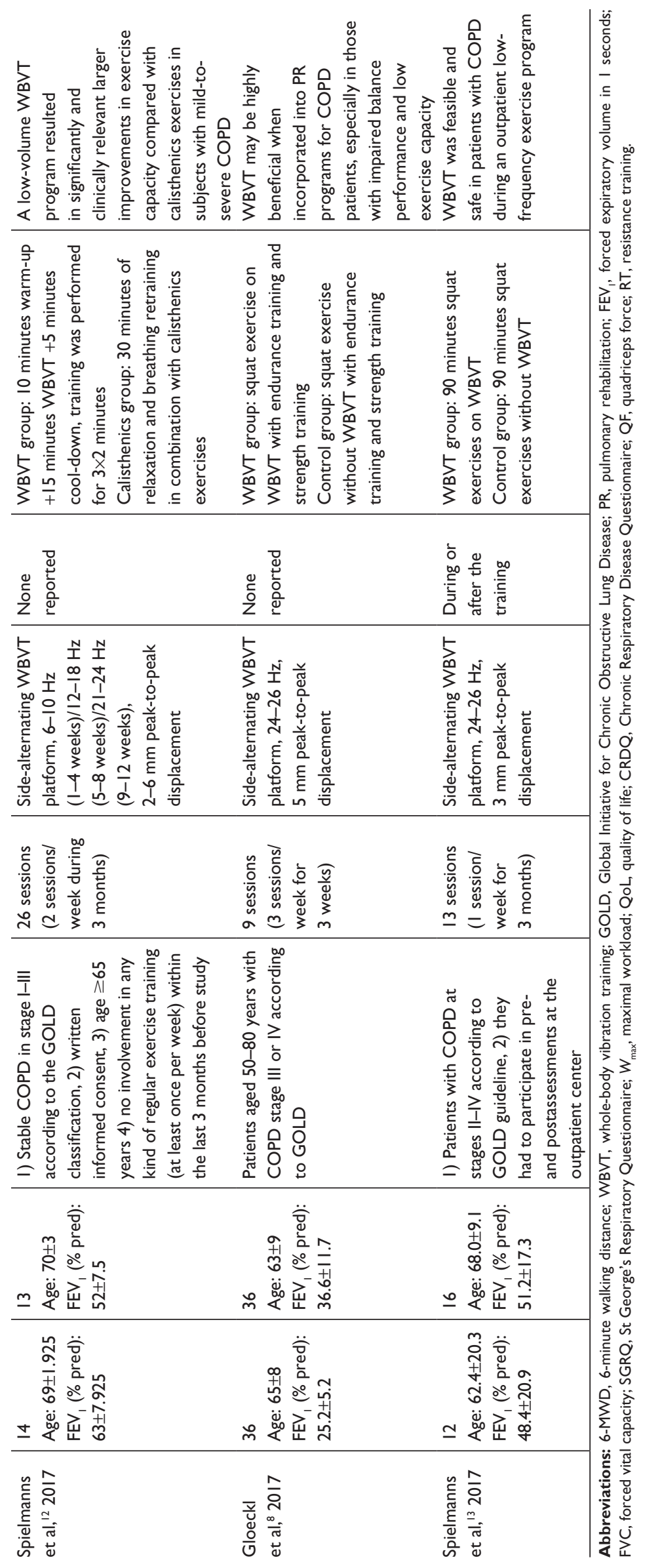


Table 2 Risk of bias assessment

\begin{tabular}{|c|c|c|c|c|c|c|c|}
\hline & $\begin{array}{l}\text { Sequence } \\
\text { generation }\end{array}$ & $\begin{array}{l}\text { Concealment } \\
\text { of allocation }\end{array}$ & $\begin{array}{l}\text { Blinding of } \\
\text { participants and } \\
\text { personnel }\end{array}$ & $\begin{array}{l}\text { Blinding of } \\
\text { outcome } \\
\text { assessors }\end{array}$ & $\begin{array}{l}\text { Incomplete } \\
\text { outcome data } \\
\text { addressed }\end{array}$ & $\begin{array}{l}\text { Free of } \\
\text { selective } \\
\text { reporting }\end{array}$ & $\begin{array}{l}\text { Other } \\
\text { bias }\end{array}$ \\
\hline Gloeckl et al,7 2012 & Low & Low & Low & Low & Low & Low & Low \\
\hline Pleguezuelos et al,,$^{10} 2013$ & Low & Unclear & Low & Low & Low & Low & Low \\
\hline Greulich et al, ${ }^{9} 2014$ & Low & Low & Low & Low & Low & Low & Low \\
\hline Braz Júnior et al, ${ }^{6} 2015$ & Low & Low & Low & Low & Low & Low & Low \\
\hline Salhi et al," 2015 & Low & Low & Unclear & Unclear & Low & Low & Low \\
\hline Spielmanns et al, ${ }^{12} 2016$ & Low & Unclear & Unclear & Unclear & Low & Low & Low \\
\hline Gloeckl et al, ${ }^{8} 2017$ & Low & Low & Low & Low & Low & Low & Low \\
\hline Spielmanns et al, ${ }^{13} 2017$ & Low & Low & Low & Low & Low & Low & Low \\
\hline
\end{tabular}

\section{Sensitivity analysis and publication bias}

We did not find any outcomes that were outside of the estimated range from derived forest plots. No significant outcomes were found in sensitivity analyses. No significant publication biases were identified by Begg's test and visual inspection of the funnel plot.

\section{Assessment of functional exercise capacity}

6-MWD and the change of 6-MWD

6-MWD and its variation- change of 6-MWD were reported in all eight trials, as shown in Figure 3. The results showed a significantly increased 6-MWD result (WMD: 62.14, 95\% CI: 48.12-76.16, $P<0.001)$ and the change of 6-MWD (WMD: 42.33, 95\% CI: 15.21-69.45, $P=0.002$ ) in WBVT group compared with control group, which indicated that WBVT led to significant improvements in functional exercise capacity. We found no significant discoveries in subgroup analyses. Side-alternating vibration was applied in five trials, ${ }^{7-9,12,13}$ in which 6-MWD did not show significant differences between two groups (WMD: 38.91, 95\% CI: -1.11 to $78.92, P=0.11$ ), but the change of 6-MWD (WMD: 43.73, 95\% CI: 14.84-72.62, $P=0.003$ ) increased above the minimal clinically important difference (MCID) of $35 \mathrm{~m} .{ }^{15}$ Vertical vibration was applied in the remaining three trials, ${ }^{6,10,11}$ but patients enrolled in these trials did not show any improvement in their 6-MWD (WMD: 38.66, 95\% CI: -47.22 to $125.54, P=0.38$ ) and change of 6-MWD (WMD: $40.51,95 \% \mathrm{CI}:-40.49$ to $121.52, P=0.33$ ) significantly.

\section{6-MWD (\% predicated) and change of 6-MWD}

(\% predicated)

Three studies ${ }^{7,11,12}$ focused on 6-MWD (\% predicated) to assess the functional exercise capacity. Compared with control group, WBVT group did not show a significantly increased 6-MWD (\% predicated) (WMD: 4.21, 95\% CI: -8.42 to $16.84, P=0.51)$ and the change of $6-\mathrm{MWD}$ (\% predicated) (WMD: 4.87, 95\% CI: -5.19 to $14.93, P=0.34$ ).

\section{SST and change of SST}

A total of five studies ${ }^{7-9,12,13}$ reported SST and the change of SST. The analysis of SST and the change of SST are presented in Figures 3 and 4. WBVT group decreased the change of SST significantly compared with control group (WMD: -2.07 seconds, $95 \% \mathrm{CI}:-4.09$ to $-0.05, P=0.04$ ). However, no significant outcome was found between the two groups regarding the SST (WMD: -1.45 seconds, $95 \% \mathrm{CI}:-3.71$ to $0.80, P=0.21)$. Side-alternating vibration was applied in all the five trials, and it decreased the change of SST above the MCID of 1.7 seconds. ${ }^{16}$

\section{Assessment of pulmonary function}

$\mathrm{FEV}_{1}$ (\% predicated) and change of FEV ,

(\% predicated)

With analysis from three included studies, ${ }^{7,9,13}$ no significant difference was found between the two groups concerning $\mathrm{FEV}_{1}$ (\% predicated) (WMD: $-0.95,95 \%$ CI: -5.50 to $3.60, P=0.68$ ), which can be seen in Figure 4, and the change of $\mathrm{FEV}_{1}(\%$ predicated) (WMD: $-1.83,95 \% \mathrm{CI}:-4.53$ to $0.87, P=0.18$ ).

\section{Assessment of quality of life SGRQ and change of SGRQ}

The change of SGRQ and SGRQ scores were reported in three trials. ${ }^{6,9,12}$ Compared with control group, WBVT decreased the change of SGRQ score (WMD: -6.65 points, 95\% CI: -10.52 to $-2.78, P<0.001)$. However, as is shown in Figure 4, no significant difference was found between the two groups regarding SGRQ score (WMD: -5.79 points, 95\% CI: -13.16 to $1.58, P=0.12$ ).

\section{CAT and change of CAT}

With analysis from three included trials,,${ }^{9,12,13}$ no significant difference was found between the two groups regarding 
A

Random sequence generation (selection bias)

Allocation concealment (selection bias)

Blinding of participants and personnel (performance bias)

Blinding of outcome assessment (detection bias)

Incomplete outcome data (attrition bias)

Selective reporting (reporting bias)

Other bias
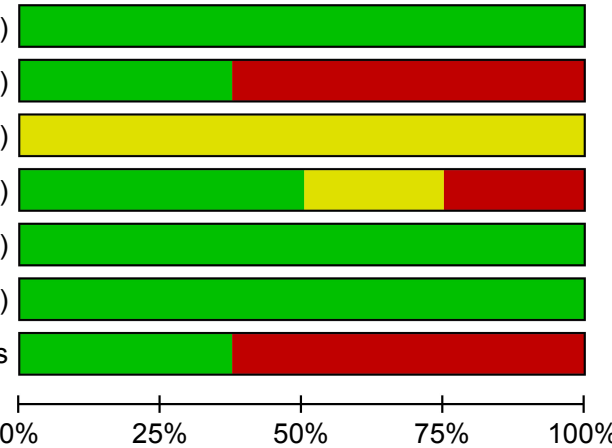

Low risk of bias $\square$ Unclear risk of bias

High risk of bias

B

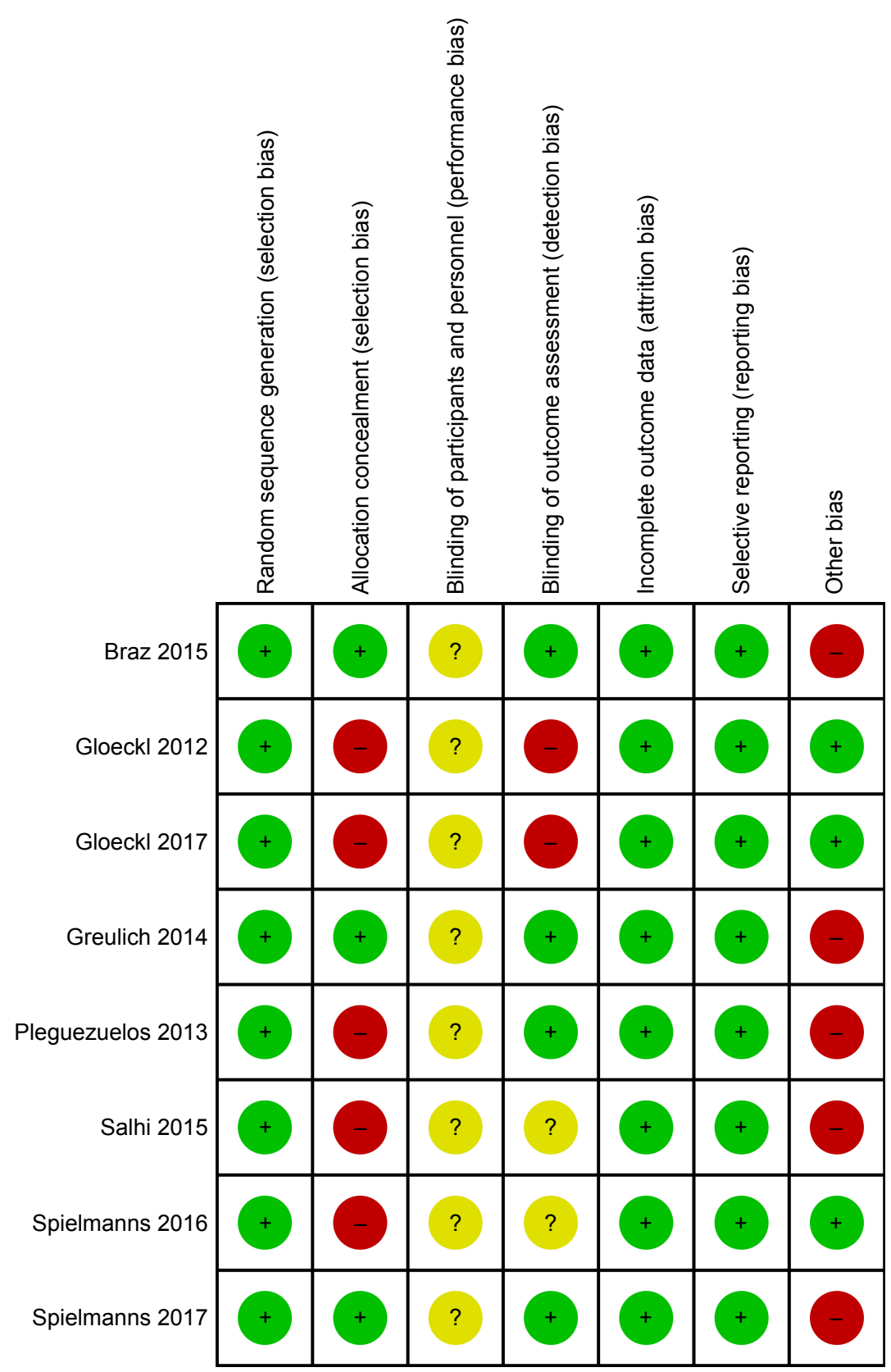

Figure 2 Assessment of risk of bias.

Notes: (A) Graph of the risk of bias for the included studies, (B) graph of the risk of bias summary for the included studies. 
A

\begin{tabular}{|c|c|c|c|c|c|c|c|c|c|c|c|c|}
\hline $\begin{array}{l}\text { Study or } \\
\text { subgroup }\end{array}$ & $\begin{array}{l}\text { WBVT } \\
\text { Mean }\end{array}$ & SD & Total & $\begin{array}{l}\text { Control } \\
\text { Mean }\end{array}$ & SD & Total & $\begin{array}{l}\text { Weight } \\
\text { (\%) }\end{array}$ & $\begin{array}{l}\text { Mean difference IV, } \\
\text { random, } 95 \% \mathrm{Cl}\end{array}$ & \multicolumn{4}{|c|}{$\begin{array}{l}\text { Mean difference IV, } \\
\text { random, } 95 \% \mathrm{Cl}\end{array}$} \\
\hline Braz 2015 & 64.18 & 103.36 & 11 & -14.73 & 113 & 11 & 5.8 & $78.91(-11.59,169.41)$ & & & & \\
\hline Gloeckl 2012 & 64 & 59.1 & 36 & 37.3 & 52.2 & 36 & 14.0 & $26.76(0.94,52.46)$ & & & - & \\
\hline Greulich 2014 & 95.55 & 76.29 & 20 & -6.13 & 81.65 & 20 & 10.5 & $101.68(52.71,150.65)$ & & & & \\
\hline Pleguezuelos 2013 & 81.2 & 9.2 & 26 & 4 & 34.22 & 25 & 15.4 & $77.20(63.33,91.07)$ & & & - & \\
\hline Salhi 2015 & 35 & 45.93 & 26 & 60 & 61.48 & 25 & 13.4 & $-25.00(-54.84,4.87)$ & & & & \\
\hline Spielmanns 2016 & 105 & 65.19 & 14 & 15 & 17.78 & 13 & 12.6 & $90.00(54.51,125.49)$ & & & $\longrightarrow$ & \\
\hline Spielmanns 2017 & 7 & 55 & 12 & 9 & 45 & 16 & 12.2 & $-2.00(-40.14,36.14)$ & & & & \\
\hline \multirow{2}{*}{\multicolumn{9}{|c|}{$\begin{array}{l}\text { Total }(95 \% \text { CI) } \\
\text { Heterogeneity: } \tau^{2}=1,192.00 ; \chi^{2}=92.40, d f=7(P<0.00001) ; I^{2}=92 \%\end{array}$}} & & & & \\
\hline & & & & & & & & & & & & \\
\hline \multicolumn{9}{|c|}{ Test for overall effect: $Z=3.06(P=0.002)$} & -200 & -100 & 100 & 200 \\
\hline
\end{tabular}

B

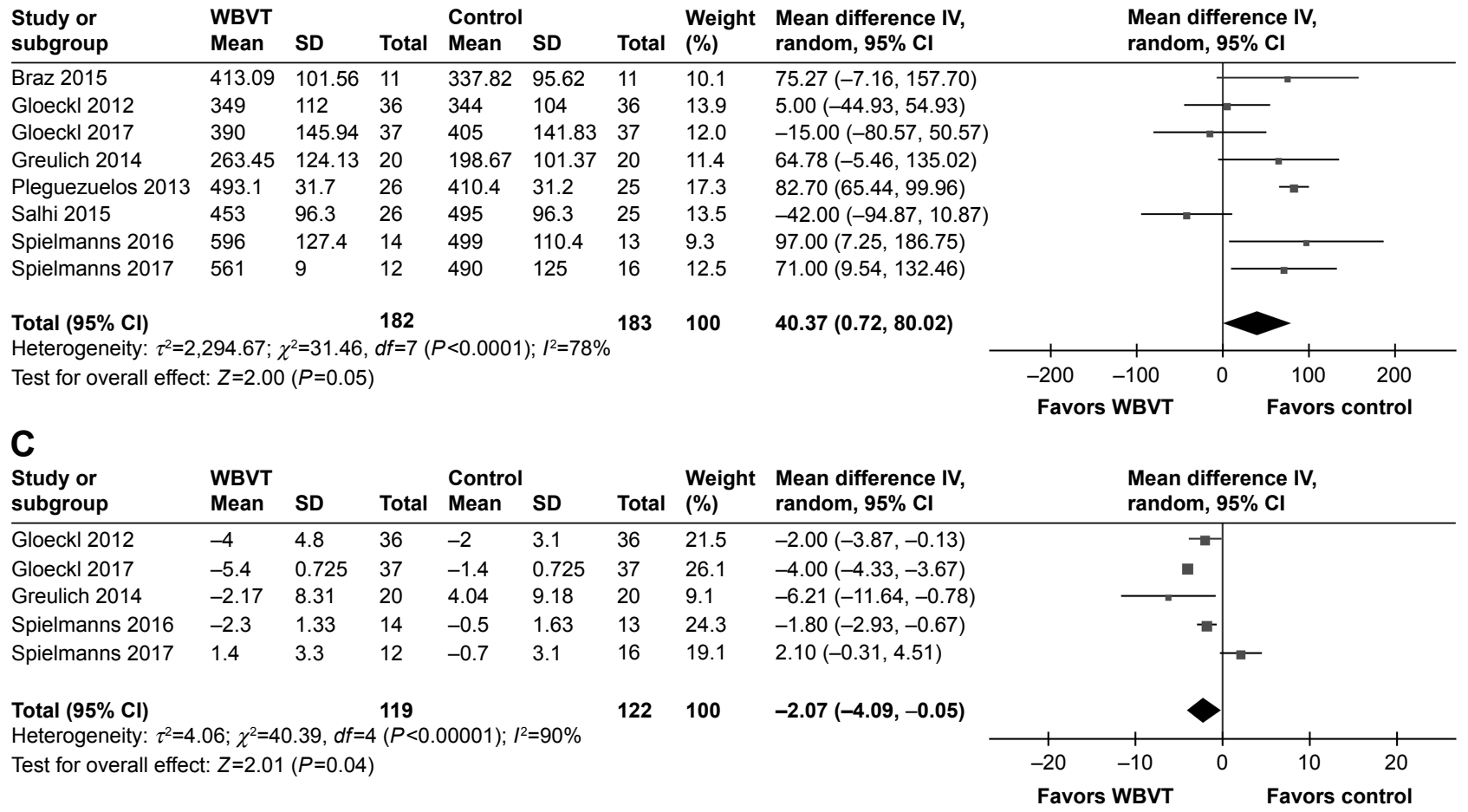

Figure 3 Meta-analysis of the effect of WBVT or control group on (A) change of 6-MWD; (B) 6-MWD; (C) change of SST.

Abbreviations: 6-MWD, 6-minute walking distance; SST, sit-to-stand test; WBVT, whole-body vibration training.

the CAT score (WMD: 1.35 points, $95 \%$ CI: -1.56 to 4.27 , $P=0.36$ ) and the change of CAT score (WMD: -0.29 points, $95 \%$ CI: -3.27 to $2.69, P=0.85$ ).

\section{CRQ and change of CRQ}

Change of CRQ and CRQ were reported in two trials; ${ }^{7,11}$ however, no significant difference was found between the two groups regarding the CRQ (WMD: $0.95,95 \% \mathrm{CI}$ : -0.64 to $2.54, P=0.24$ ) and the change of CRQ (WMD: 0.27 points, $95 \% \mathrm{CI}:-1.41$ to $1.96, P=0.75$ ).

\section{Change of Berg scale and Berg scale}

Based on the analysis of the two included studies, ${ }^{10,12}$ we found no significant difference between WBVT group and control group regarding the Berg scale (WMD: 0.27 points, 95\% CI: -1.37 to $1.92, P=0.74)$ and the change of Berg scale (WMD: 1.49 points, $95 \% \mathrm{CI}:-3.10$ to $6.09, P=0.52$ ).

\section{Discussion}

Our study included eight RCTs to assess the efficacy and safety of WBVT in improving functional exercise capacity, pulmonary function, and quality of life in COPD patients. The present systematic review showed that WBVT had beneficial effects on functional exercise capacity, which was mainly measured by 6-MWD and change of 6-MWD. However, limited evidence suggested that WBVT might enhance pulmonary function and quality of life in COPD patients regarding the change of $\mathrm{FEV}_{1}$ (\% predicated) and SGRQ score. 


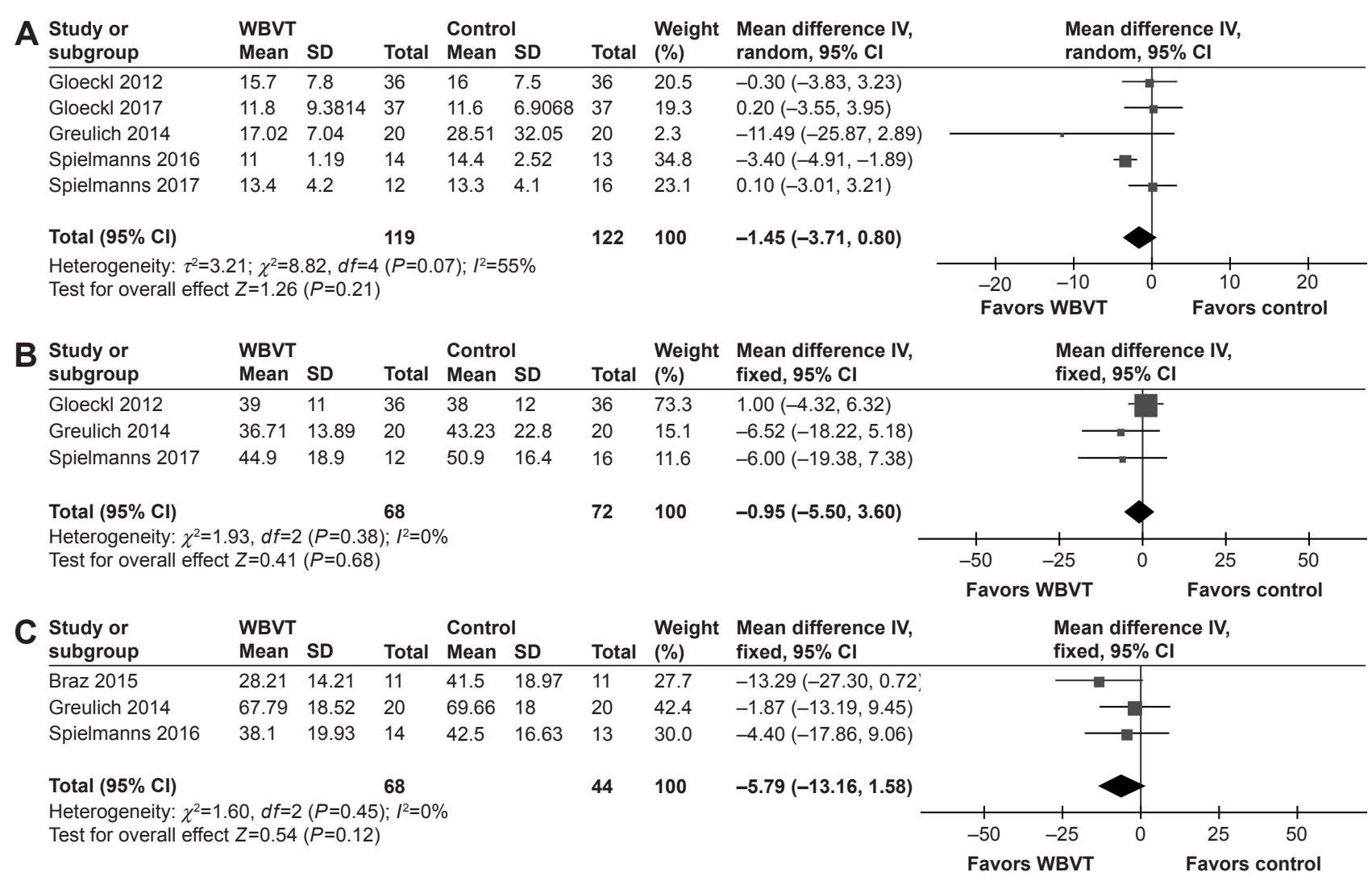

Figure 4 Meta-analysis of the effect of WBVT or control on (A) SST; (B) FEV (\% predicated); (C) SGRQ.

Abbreviations: FEV , forced expiratory volume in the first second; SGRQ, St George's Respiratory Questionnaire; SST, sit-to-stand test; WBVT, whole-body vibration training.

Some previous systematic reviews ${ }^{17-19}$ focused on the question of whether WBVT was better than conventional training in pulmonary function and exercise capacity. Gloeckl et a ${ }^{17}$ performed a systematic review in 2015 which included six studies. ${ }^{6,7,9,10,20,21}$ However, we found that two of six included studies ${ }^{20,21}$ might not be RCTs. Yang et $\mathrm{al}^{18}$ and Cardim et al ${ }^{19}$ performed similar systematic reviews in 2016. The two systematic reviews performed by them included the same four RCTs. ${ }^{6,9,10,22}$ They both drew the conclusion that WBVT may improve functional exercise capacity of patients with COPD.

Compared with the previous systematic reviews, our present systematic review has some advantages. First, we included eight RCTs based on strict inclusion criteria, with a total of 365 patients enrolled. Second, we analyzed more outcomes, such as the change of 6-MWD, to measure the differences. Last but not the least, we performed more subgroup analyses, leading to new significant findings such as the fact that the type of WBVT influences the effects of training. These are the main advantages of this meta-analysis.

To clarify the effects of WBVT on functional exercise capacity, we used 6-MWD, the change of 6-MWD, 6-MWD
(\% predicated), the change of 6-MWD (\% predicated), and SST and the change of SST. 6-MWD and the change of 6-MWD were the most important outcomes showing significant differences. Patients enrolled in this meta-analysis increased their 6-MWD above the MCID of $35 \mathrm{~m} .{ }^{15}$ Similar results were observed for the SST and SGRQ; the time needed for SST decreased above the MCID of 1.7 seconds. ${ }^{16} \mathrm{FEV}_{1}$ (\% predicated) and the change of $\mathrm{FEV}_{1}$ (\% predicated) were used to measure the pulmonary function of COPD patients. Our results showed that no significant difference was found between the two groups, which indicated that WBVT provided limited benefits to pulmonary function. The change of SGRQ, SGRQ; the change of CAT, CAT; the change of CRQ, CRQ; and the change of Berg scale, Berg scale were used to measure the quality of life in COPD patients. Only the change of SGRQ decreased above the MCID of $4 .{ }^{23}$ Considering a reduction in quality of evidence due to the risk of bias, the inaccuracy and inconsistency, it is hard to draw the conclusion that WBVT has benefits on the quality of life in COPD patients.

The underlying mechanisms by which WBVT improves muscle function are incompletely clarified. During WBVT, 
the vibrations are transmitted to the muscle group through the limbs, which motivates the activation of the active muscle and improves the biological activity of the high threshold motion unit, leading to the participation of the muscle group in the sports unit. ${ }^{24}$ The effects of WBVT therapy can be divided into two types: direct biological effect and indirect biological effect. Direct effects derive from the mechanical vibration waves to the muscle group. Indirect effects are connected to the reflex and regulation of nerve and body fluids caused by mechanical vibration waves. The mechanism by which WBVT can improve functional exercise capacity of people with CODP might be closely related to the following factors.

First, WBVT led to improvements in neuromuscular activation. The most common mechanism to explain WBVTinduced reflex muscular activity is the tonic vibration reflex. ${ }^{25}$ Different from the voluntary muscle control in traditional resistance training, the muscle contractions during WBVT are stimulated by stretch reflexes. ${ }^{26}$ The length of the muscle spindle generally increases and afferents from Golgi tendon organs become more sensitive to vibration, ${ }^{25}$ which is seen as an effective measurement of tendon elongation and a surrogate of force. ${ }^{27}$ People feel an experience of movement during the tonic vibration reflex, ${ }^{28}$ in which the discharge from Golgi tendon organs increases, which means better muscle force. ${ }^{25}$ Furthermore, specific increases in ATP turnover and a generation of excess heat follow vibrations to fiber preparations, ${ }^{24}$ which is complied with increased skin and muscle perfusion. ${ }^{29-31}$

Second, WBVT improves blood circulation in people with COPD. At present, the relevant literature shows that after WBVT, the peripheral blood circulation of the body increases by nearly $14 \% .{ }^{32}$ WBVT can increase the amount of vascular endothelial growth factor and endothelia in blood, thus accelerating blood vessel formation. ${ }^{33}$ WBVT can also effectively improve the dilatation capacity of arterioles, ${ }^{34}$ the speed of the blood flow, the flexibility and coordination of the muscle and also reduce the threshold of the excitability of the muscle spindle at the same time,${ }^{35}$ which shows important physiological significance to indirectly improve the exercise capacity of people with COPD.

Furthermore, the effects of WBVT on the respiratory system are mostly focused on alleviating the clinical symptoms of lung diseases. WBVT can effectively improve the clinical symptoms of patients with COPD, which is mainly related to the strengthened contraction force of the respiratory muscles, which further stimulates the respiratory function, improves the ratio of ventilation, blood flow, and the respiratory function. ${ }^{7,10}$ Taking all related factors together, WBVT can effectively improve the muscle performance.
Our results showed heterogeneity across included studies. We concluded the possible reasons of heterogeneity as follows. First, the severity of participants' state of COPD ranged from stable to severe, so different stages of COPD could result in the heterogeneity. Second, the various assessments of effects of WBVT could contribute to the heterogeneity. Furthermore, inhomogeneous study protocols and settings (study period, WBVT device, WBVT parameters, exercises performed on the WBVT platform, age of patients) made the heterogeneity inevitable.

To clarify the origin of heterogeneity, we tried to perform subgroup analyses. The type of vibration was an important factor related to the effects of WBVT. Side-alternating vibration increased the change of 6-MWD and decreased the change of SST above the MCID, but vertical vibration did not lead to any significant difference. Subgroup analyses showed that side-alternating vibration may be better than vertical vibration. Also, WBVT had different effects on patients at different age, but not those of different ethnicity. Besides, people with stable COPD benefited more than people with severe COPD. However, the limited sample made it hard to decide whether longer training period would have greater effects.

The first potential limitation of this present systematic review was the different training protocols and inconsistent control group interventions used among studies, which contributed mostly to the heterogeneity of the outcomes. Another limitation is that due to limited intention-to-treat data reported, our primary analysis was not according to intention-to-treat analyses, which may bias our results.

\section{Conclusion}

In conclusion, the current evidence indicates that WBVT is a safe modality that has beneficial effects on functional exercise capacity but limited effects on pulmonary function and quality of life of COPD patients. More high-quality RCTs are needed to confirm this.

\section{Acknowledgment}

We give sincere thanks to Hong Xie for her advice with respect to the English language editing of this manuscript.

\section{Author contributions}

FL, JZ, and LP conceived and designed the study. FL provided administrative support. Provision of study materials or patients was due to JZ. JZ and LP helped in the collection and assembly of data. JZ, LP, NC, ZW, CW, YH, ML, and HL were involved with data analysis. FL, JZ, LP, NC, ZW, $\mathrm{CW}, \mathrm{YH}, \mathrm{ML}$, and HL were involved in manuscript writing and final approval of the paper. All authors contributed 
toward data analysis, drafting and critically revising the paper and agree to be accountable for all aspects of the work.

\section{Disclosure}

The authors report no conflicts of interest in this work.

\section{References}

1. Rauch F, Sievanen H, International Society of Musculoskeletal and Neuronal Interactions. Reporting whole-body vibration intervention studies: recommendations of the International Society of Musculoskeletal and Neuronal Interactions. J Musculoskelet Neuronal Interact. 2010;10(3):193-198.

2. Gloeckl R, Marinov B, Pitta F. Practical recommendations for exercise training in patients with COPD. Eur Respir Rev. 2013;22(128) $178-186$

3. Roelants M, Delecluse C, Verschueren SM. Whole-body-vibration training increases knee-extension strength and speed of movement in older women. J Am Geriatr Soc. 2004;52(6):901-908.

4. Spruit MA, Singh SJ, Garvey C, et al. An official American Thoracic Society/European Respiratory Society statement: key concepts and advances in pulmonary rehabilitation. [Erratum appears in Am J Respir Crit Care Med. 2014;189(12):1570]. Am J Respir Crit Care Med. 2013;188(8):15.

5. Armijo-Olivo S, Stiles CR, Hagen NA, Biondo PD, Cummings GG. Assessment of study quality for systematic reviews: a comparison of the Cochrane Collaboration Risk of Bias Tool and the Effective Public Health Practice Project Quality Assessment Tool: methodological research. J Eval Clin Pract. 2012;18(1):12-18.

6. Braz Júnior DS, Dornelas de Andrade A, Teixeira AS, Cavalcanti CA, Morais AB, Marinho PE. Whole-body vibration improves functional capacity and quality of life in patients with severe chronic obstructive pulmonary disease (COPD): a pilot study. Int J Chron Obstruct Pulmon Dis. 2015;10:125-132.

7. Gloeckl R, Heinzelmann I, Baeuerle S, et al. Effects of whole body vibration in patients with chronic obstructive pulmonary disease - a randomized controlled trial. Respir Med. 2012;106(1):75-83.

8. Gloeckl R, Jarosch I, Bengsch U, et al. What's the secret behind the benefits of whole-body vibration training in patients with COPD? A randomized, controlled trial. Respir Med. 2017;126:17-24.

9. Greulich T, Nell C, Koepke J, et al. Benefits of whole body vibration training in patients hospitalised for COPD exacerbations - a randomized clinical trial. BMC Pulm Med. 2014;14:60.

10. Pleguezuelos E, Pérez ME, Guirao L, et al. Effects of whole body vibration training in patients with severe chronic obstructive pulmonary disease. Respirology. 2013;18(6):1028-1034.

11. Salhi B, Malfait TJ, van Maele G, Joos G, van Meerbeeck JP, Derom E. Effects of whole body vibration in patients with COPD. COPD. 2015; 12(5):525-532.

12. Spielmanns M, Boeselt T, Gloeckl R, et al. Low-volume whole-body vibration training improves exercise capacity in subjects with mild to severe COPD. Respir Care. 2017;62(3):315-323.

13. Spielmanns M, Gloeckl R, Gropp JM, et al. Whole-body vibration training during a low frequency outpatient exercise training program in chronic obstructive pulmonary disease patients: a randomized, controlled trial. J Clin Med Res. 2017;9(5):396-402.

14. Salhi B, van Meerbeeck J, Joos GF, Derom E. Effects of whole body vibration in patients with COPD: a randomized study. Am J Respir Crit Care Med. 2011;2011:183.

15. Regueiro EM, Burtin C, Baten P, et al. The minimal important difference of the pulmonary functional status and dyspnea questionnaire in patients with severe chronic obstructive pulmonary disease. Respir Res. 2013;14:58.

16. Jones SE, Kon SS, Canavan JL, et al. The five-repetition sit-to-stand test as a functional outcome measure in COPD. Thorax. 2013;68(11) 1015-1020.
17. Gloeckl R, Heinzelmann I, Kenn K. Whole body vibration training in patients with COPD: A systematic review. Chron Respir Dis. 2015; 12(3):212-221.

18. Yang X, Zhou Y, Wang P, He C, He H. Effects of whole body vibration on pulmonary function, functional exercise capacity and quality of life in people with chronic obstructive pulmonary disease: a systematic review. Clin Rehabil. 2016;30(5):419-431.

19. Cardim AB, Marinho PE, Nascimento JF, Fuzari HK, Dornelas de Andrade A. Does whole-body vibration improve the functional exercise capacity of subjects with COPD? A Meta-Analysis. Respir Care. 2016;61(11):1552-1559.

20. Furness T, Joseph C, Welsh L, Naughton G, Lorenzen C. Whole-body vibration as a mode of dyspnoea free physical activity: a communitybased proof-of-concept trial. BMC Res Notes. 2013;6(1):452.

21. Furness T, Joseph C, Naughton G, Welsh L, Lorenzen C. Benefits of whole-body vibration to people with COPD: a community-based efficacy trial. BMC Pulm Med. 2014;14:38.

22. Gloeckl R, Halle M, Kenn K. Interval versus continuous training in lung transplant candidates: a randomized trial. J Heart Lung Transplant. 2012;31(9):934-941.

23. Welling JB, Hartman JE, Ten Hacken NH, Klooster K, Slebos DJ. The minimal important difference for the St George's Respiratory Questionnaire in patients with severe COPD. Eur Respir J. 2015;46(6): 1598-1604.

24. Robinson JM, Wang Y, Kerrick WG, Kawai R, Cheung HC. Activation of striated muscle: nearest-neighbor regulatory-unit and cross-bridge influence on myofilament kinetics. J Mol Biol. 2002;322(5):1065-1088.

25. Burke D, Schiller HH. Discharge pattern of single motor units in the tonic vibration reflex of human triceps surae. J Neurol Neurosurg Psychiatry. 1976;39(8):729-741.

26. Cardinale M, Lim J. Electromyography activity of vastus lateralis muscle during whole-body vibrations of different frequencies. $J$ Strength Cond Res. 2003;17(3):621-624.

27. Treloar AE, Boynton RE, Behn BG, Brown BW. Variation of the human menstrual cycle through reproductive life. Int J Fertil. 1967;12(1 Pt 2): $77-126$.

28. Goodwin P. Personal involvement. Nurs Times. 1972;68(52):1660.

29. Lohman EB, Sackiriyas KS, Bains GS, et al. A comparison of whole body vibration and moist heat on lower extremity skin temperature and skin blood flow in healthy older individuals. Med Sci Monit. 2012; 18(7):CR415-CR424.

30. Maloney-Hinds C, Petrofsky JS, Zimmerman G. The effect of $30 \mathrm{~Hz}$ vs. $50 \mathrm{~Hz}$ passive vibration and duration of vibration on skin blood flow in the arm. Med Sci Monit. 2008;14(3):Cr112-Cr116.

31. Rittweger J, Rauch F. What is new in musculo-skeletal interactions. J Musculoskelet Neuronal Interact. 2000;1(2):171-176.

32. Button C, Anderson N, Bradford C, Cotter JD, Ainslie PN. The effect of multidirectional mechanical vibration on peripheral circulation of humans. Clin Physiol Funct Imaging. 2007;27(4):211-216.

33. Suhr F, Brixius K, de Marées M, et al. Effects of short-term vibration and hypoxia during high-intensity cycling exercise on circulating levels of angiogenic regulators in humans. J Appl Physiol. 2007;103(2): 474-483.

34. Rosenberger A, Liphardt AM, Bargmann A, et al. EMG and heart rate responses decline within 5 days of daily whole-body vibration training with squatting. PLoS One. 2014;9(6):e99060.

35. Kerschan-Schindl K, Grampp S, Henk C, et al. Whole-body vibration exercise leads to alterations in muscle blood volume. Clin Physiol. 2001; 21(3):377-382.

36. Moher D, Liberati A, Tetzlaff J, Altman DG; The PRISMA Group. Preferred Reporting Items for Systematic Reviews and Meta-Analyses: The PRISMA Statement. PLoS Med. 2009;6(7):e1000097. 


\section{Publish your work in this journal}

The International Journal of COPD is an international, peer-reviewed journal of therapeutics and pharmacology focusing on concise rapid reporting of clinical studies and reviews in COPD. Special focus is given to the pathophysiological processes underlying the disease, intervention programs, patient focused education, and self management protocols.

This journal is indexed on PubMed Central, MedLine and CAS. The manuscript management system is completely online and includes a very quick and fair peer-review system, which is all easy to use. Visit http://www.dovepress.com/testimonials.php to read real quotes from published authors.

Submit your manuscript here: http://www.dovepress.com/international-journal-of-chronic-obstructive-pulmonary-disease-journal 